\title{
ON A COEFFICIENT PROBLEM FOR BI-UNIVALENT FUNCTIONS
}

\section{LEWIN}

1. Introduction. Let $S$ be the family of analytic functions:

$$
f(z)=z+a_{2} z^{2}+\cdots,
$$

which are regular and univalent in $|z|<1$. Let $f^{-1}(z)$ be the inverse function of $f(z)$. We have:

$$
f^{-1}(z)=z-a_{2} z^{2}+\left(2 a_{2}^{2}-a_{3}\right) z^{3}+\cdots .
$$

The function $f(z)$ will be called bi-univalent if both $f(z)$ and $f^{-1}(z)$ are univalent in $|z|<1 ; f(z)$ will be said to belong to $\sigma$ iff (i) $f(z) \in S$ and (ii) there exists a function $g(z) \in S$ such that $f(g(z))=g(f(z))=z$ in some neighborhood of the origin.

Z. Nehari remarked ${ }^{1}$ that if $\phi(z)=\phi_{1} z+\phi_{2} z^{2}+\cdots$ and $\psi(z)$ $=\psi_{1} z+\psi_{2} z^{2}+\cdots$, with $\psi_{1}=\phi_{1}$, are two functions mapping the open unit circle onto a schlicht domain containing the open unit circle, then the function

$$
f(z)=\phi\left[\psi^{-1}(z)\right]
$$

is bi-univalent. It will belong to $\sigma$ if both $f(z)$ and $f^{-1}(z)$ are regular in $|z|<1$.

It is not clear whether all functions of $\sigma$ are representable in the form (3). If they are, the representation may not be unique. The function $f(z)$ in (3) will belong to $\sigma$ even though $\phi(z)$ or $\psi(z)$ or both have poles in $|z|<1$, on condition that if $z=p_{\phi}$ and $z=p_{\psi}$ are the respective poles, the following two inequalities be satisfied:

$$
\left|\phi\left(p_{\psi}\right)\right| \geqq 1 \text { and }\left|\psi\left(p_{\phi}\right)\right| \geqq 1 \text {. }
$$

It is also not clear whether all functions of $\sigma$, even if they are of the form (3), can be represented by functions $\phi(z)$ and $\psi(z)$ which are both regular in $|z|<1$.

It is the purpose of this paper to obtain the following theorem: For $f(z) \in \sigma,\left|a_{2}\right|<1.51$.

2. Application of the area-theorem. The area-theorem applied to the function $f(z) \in S$ in (1) yields:

Received by the editors February 20, 1966 and, in revised form, July 16, 1966.

${ }^{1}$ Oral communication. 


$$
\sum_{p=1}^{\infty} p\left|c_{p}\right|^{2} \leqq 1
$$

where

$$
\frac{1}{f(z)}=z^{-1}+\sum_{p=0}^{\infty} c_{p} z^{p}
$$

Let $f_{n}(z)=\left[f\left(z^{n}\right)\right]^{(1 / n)}$ for integral $n \geqq 1$. If $f(z) \in S$, then $f_{n}(z) \in S$. It follows that if $f(z) \in \sigma$ then $f_{n}(z) \in \sigma$. Applying inequality (3) to $f_{n}(z)$ we obtain

$$
(n-1)\left|a_{2}\right|^{2}+(2 n-1)\left|a_{3}-((n+1) / 2 n) a_{2}^{2}\right|^{2} \leqq n^{2}
$$

and hence:

$$
\left|a_{3}-\frac{n+1}{2 n} a_{2}^{2}\right| \leqq\left(\frac{n^{2}-(n-1)\left|a_{2}\right|^{2}}{2 n-1}\right)^{1 / 2} .
$$

Applying (4) to the function $f_{n}^{-1}(z)$ we get:

$$
\left|a_{3}-\frac{3 n-1}{2 n} a_{2}^{2}\right| \leqq\left(\frac{n^{2}-(n-1)\left|a_{2}\right|^{2}}{2 n-1}\right)^{1 / 2} \text {. }
$$

Inequalities (5) and (6) imply:

$$
\frac{(n-1)}{2 n}\left|a_{2}\right|^{2} \leqq\left(\frac{n^{2}-(n-1)\left|a_{2}\right|^{2}}{2 n-1}\right)^{1 / 2} .
$$

By squaring both sides a quadratic inequality for $\left|a_{2}\right|^{2}$ is obtained, whence:

$$
\left|a_{2}\right|^{2} \leqq \frac{2 n^{2}}{(n-1)\left((2 n)^{1 / 2}+1\right)} .
$$

For $n=2,3$ and 4 the right-hand side of (7) takes the values 2.67, 2.61 and 2.78 respectively. We thus get the best result for $n=3$, so that:

$$
\left|a_{2}\right|^{2}<2.61
$$

or:

$$
\left|a_{2}\right|<1.62,
$$

This bound can be improved by the use of Grunsky's inequalities.

3. The $l$-sequence $\left\{l_{k}\right\}$. We use the sequence $\left\{l_{k}\right\}$ introduced by E. Jabotinsky [3]. Let $f(s, z)$ be the $s$-iterate of

$$
f(z)=z+a_{2} z^{2}+\cdots \cdot
$$


That is, let $f(s, z)$ be defined for integer $s$ by:

Then:

$$
f(1, z)=f(z) \text { and } f(s, z)=f[(s-1), f(z)] .
$$

$$
f(s, z)=z+\sum_{p=2}^{\infty} a_{p}(s) z^{p},
$$

where $a_{p}(s)$ is a polynomial of degree $(p-1)$ in $s$. We define:

$$
l_{k}=\left.\frac{d}{d s} a_{k-1}(s)\right|_{s=0} .
$$

The sequences $\left\{a_{i}\right\}$ and $\left\{l_{k}\right\}$ determine each other uniquely. In particular:

$$
a_{2}=l_{1}, \quad a_{3}=l_{2}+l_{1}^{2}, \quad a_{4}=l_{3}+\frac{5}{2} l_{1} l_{2}+l_{1}^{3}
$$

also:

$$
l_{1}=a_{2}, \quad l_{2}=a_{3}-a_{2}^{2}, \quad l_{3}=a_{4}-\frac{5}{2} a_{2} a_{3}+\frac{3}{2} a_{2}^{3} .
$$

The sequence $\left\{l_{k}\right\}$ has the following two properties: then:

(A) Let $\left\{\lambda_{k}\right\}$ be the $l$-sequence of the function $f^{-1}(z)=f(-1, z)$,

$$
\lambda_{k}=-l_{k} .
$$

(B) Let $\left\{l_{k}(n)\right\}$ be the $l$-sequence of the function $f_{n}(z)=\left[f\left(z^{n}\right)\right]^{1 / n}$. We have:

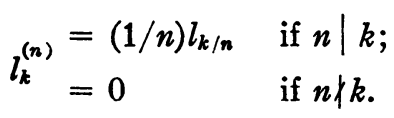

Proof of A. Let $L(z)=\sum_{k=1}^{N} l_{k} z^{k+1}$ (where $N$ is a large but otherwise arbitrary integer). $L(z)$ then defines an analytic function $f^{*}(z)$ $=z+a_{2} z^{2}+\cdots$ and $L(z)$ is the coefficient of $s$ in the Taylor expansion of $f^{*}(s, z)$ about $s=0$. The existence of such an expansion is proved in [2]. On the other hand the first $N+1$ coefficients of the respective power-series expansions of $f^{*}(z)$ and $f(z)$ coincide. (If $f(s, z)$ admits a power-series expansion in $s$, we may define $L(z)$ $=\sum_{k=1}^{\infty} l_{k} z^{k+1}$ which has then a positive radius of convergence [3], so that $f^{*}(z) \equiv f(z)$.) Let now $g^{*}(z)$ be the inverse of $f^{*}(z)$ so that

$$
g^{*}(s, z)=f^{*}(-s, z) .
$$

It follows immediately that

$$
L^{\left(o^{*}\right)}(z) \equiv-L^{\left(f^{*}\right)}(z)
$$

whence (12) holds for every $k \leqq N$. 
Since $N$ may be taken arbitrarily large, (12) holds for every $k$.

Proof of B. Define $F_{n}(z)=\left[f^{*}\left(z^{n}\right)\right]^{1 / n}$.

It is easily seen that

$$
F_{n}(s, z)=\left[f^{*}\left(s, z^{n}\right)\right]^{1 / n}
$$

Differentiating both sides with respect to $s$ gives:

$$
\frac{\partial F_{n}(s, z)}{\partial s}=\frac{1}{n} f^{*}\left(s, z^{n}\right)^{1 / n-1} \frac{\partial f^{*}\left(s, z^{n}\right)}{\partial s} .
$$

Putting now $s=0$ and noting that $f^{*}(0, z) \equiv z$, we obtain

$$
L^{(n)}(z)=\frac{1}{n} z^{1-n} L\left(z^{n}\right),
$$

or

$$
\sum_{\nu=1}^{\infty} l_{\nu}^{(n)} z^{n+1}=\frac{1}{n} z^{1-n} \sum_{k=1}^{N} l_{k}\left(z^{n}\right)^{k+1}=\sum_{k=1}^{N} \frac{1}{n} l_{k} z^{n k+1},
$$

whence, by equating coefficients, $B$ is obtained immediately.

4. Grunsky's inequalities. Grunsky [1] established a necessary and sufficient condition for the function $f(z)=z+a_{2} z^{2}+\cdots$ to be univalent in $|z|<1$. Let the numbers $g_{m n}$ be defined by:

$$
\log \frac{f(z)-f(w)}{z-w}=\log \frac{f(z)}{z}+\log \frac{f(w)}{w}+\sum_{m, n}^{\infty} g_{m n} z^{m} w^{n} .
$$

Then Grunsky's condition is:

$$
\left|\sum_{m, n=1}^{N} g_{m n} x_{m} x_{n}\right| \leqq \sum_{p=1}^{N} \frac{\left|x_{p}\right|^{2}}{p}
$$

for every choice of the complex numbers $x_{k}$ and all $N$.

In [3] the $g_{m n}$ corresponding to the function $f(z)$ are given explicitly in terms of the $l_{k}$ of that function. In particular:

$$
\begin{aligned}
& g_{11}=l_{2}, \\
& g_{12}=g_{21}=l_{3}+\frac{1}{2} l_{1} l_{2}, \\
& g_{13}=g_{31}=l_{4}+l_{1} l_{3}+\frac{1}{2} l_{2}^{2}+\frac{1}{3} l_{1}^{2} l_{2}, \\
& g_{22}=l_{4}+l_{1} l_{3}+\frac{1}{3} l_{1}^{2} l_{2}, \\
& g_{33}=l_{6}+2 l_{1} l_{5}+l_{2} l_{4}+\frac{1}{2} l_{1}^{2} l_{2}^{2}+2 l_{1}^{2} l_{4}+\frac{4}{3} l_{1} l_{2} l_{3}+\frac{1}{3} l_{2}^{3}+l_{1}^{3} l_{3}+\frac{1}{5} l_{1}^{4} l_{2} .
\end{aligned}
$$

For bi-univalent functions, Grunsky's inequalities (20) will come in pairs for every $N$, obtained from each other by replacing, according 
to (12), every $l_{k}$ by $-l_{k}$. Hence, in these inequalities, odd powers of the l's can be separated from the even powers, yielding separate inequalities, by using the fact that if $|a+b| \leqq k$ and $|a-b| \leqq k$ then $|a| \leqq k$ and $|b| \leqq k$.

5. A bound for $\left|l_{1} l_{3}\right|$. Grunsky's inequality (20) for $N=2$ becomes:

$$
\left|l_{2} x_{1}^{2}+2\left(l_{3}+\frac{1}{2} l_{1} l_{2}\right) x_{1} x_{2}+\left(l_{4}+l_{1} l_{3}+\frac{1}{3} l_{1}^{2} l_{2}\right) x_{2}^{2}\right| \leqq\left|x_{1}\right|^{2}+\frac{1}{2}\left|x_{2}\right|^{2} \text {. }
$$

Taking $x_{1}=0, x_{2}=1$ and omitting all odd powers of the $l$ 's, we get, for bi-univalent functions:

$$
\left|l_{1} l_{3}\right| \leqq \frac{1}{2} .
$$

This result can be improved for functions in $\sigma$ by the use of (8) (valid for functions of $\sigma$ only). Indeed, choosing $x_{1}=l_{1}, x_{2}=\beta>0$ and omitting all odd powers of the l's, (21) yields, for functions in $\sigma$ :

$$
\left|l_{1} l_{3}\right|\left(2 \beta+\beta^{2}\right) \leqq\left|l_{1}\right|^{2}+\frac{1}{2} \beta^{2} .
$$

Here the best result for $\left|l_{1} l_{3}\right|$, given $\left|l_{1}\right|^{2}$, is obtained by taking:

whence:

$$
\beta=\left|l_{1}\right|^{2}+\left(\left|l_{1}\right|^{4}+2\left|l_{1}\right|^{2}\right)^{1 / 2},
$$

$$
\left|l_{1} l_{3}\right| \leqq \frac{1}{\left(1+2 /\left|l_{1}\right|^{2}\right)^{1 / 2}+1} .
$$

Using the fact that $l_{1}=a_{2}$, and the bound (8) obtained by taking $n=3$ in $(7)$ :

$$
\left|l_{1} l_{3}\right| \leqq \frac{3}{3+(11+2.6)^{1 / 2}}<0.43 .
$$

We shall use this bound to improve (8).

6. An improved bound for $\left|a_{2}\right|=\left|l_{1}\right|$. Grunsky's inequality (20) for $N=3$, written for $f_{2}(z)=\left(f\left(z^{2}\right)\right)^{1 / 2}$, using (13) and taking $x_{2}=0$, is:

$$
\begin{gathered}
\left|\frac{1}{2} l_{1} x_{1}^{2}+\left(l_{2}+\frac{1}{4} l_{1}^{2}\right) x_{1} x_{3}+\left(\frac{1}{2} l_{3}+\frac{1}{4} l_{1} l_{2}+\frac{1}{24} l_{1}^{3}\right) x_{3}^{2}\right| \\
\leqq\left|x_{1}\right|^{2}+\frac{1}{3}\left|x_{3}\right|^{2},
\end{gathered}
$$

Choosing $x_{1}=l_{1}, x_{3}=\beta>0$ and omitting all even powers of the $l$ 's, (25) becomes:

$$
\left|l_{1}^{3}\left(\frac{1}{2}+\frac{1}{4} \beta+\frac{1}{24} \beta^{2}\right)+\frac{1}{2} l_{3} \beta^{2}\right| \leqq\left|l_{1}\right|^{2}+\frac{1}{3} \beta^{2},
$$

whence:

$$
\left|l_{1}\right|^{3}\left(\frac{1}{2}+\frac{1}{4} \beta+\frac{1}{24} \beta^{2}\right) \leqq\left|l_{1}\right|^{2}+\frac{1}{3} \beta^{2}+\frac{1}{2}\left|l_{3}\right| \beta^{2} .
$$


Multiplying by $\left|l_{1}\right|$ and substituting for $\left|l_{1} l_{3}\right|$ from (24) this becomes

$$
\left|l_{1}\right|^{4}\left(\frac{1}{2}+\frac{1}{4} \beta+\frac{1}{24} \beta^{2}\right)-\left|l_{1}\right|^{3}-\frac{1}{3} \beta\left|l_{1}\right|<\frac{0.43}{2} \beta^{2} .
$$

Choosing $\beta=1.5$ this yields, for functions in $\sigma$ :

$$
\left|a_{2}\right|<1.51
$$

Further improvement of both bounds (8) and (24) by the interplay of inequalities (23) and (26) yields very little. We have not succeeded in bringing down the bound in (25) to $\frac{3}{2}$.

\section{BIBLIOGRAPHY}

1. H. Grunsky, Koeffizientenbedingungen für schlicht abbildende meromorphe Funktionen, Math. Z. 45 (1939), 29-61.

4. P. Erdös and E. Jabotinsky, On analytic iteration, J. Analyse Math. 8 (19601961), 361-376.

3. E. Jabotinsky, Analytic iteration, Trans. Amer. Math. Soc. 108 (1963), 457-477.

TECHNION, HAIFA, ISRAEL 